\title{
Triangle Free Sets and Arithmetic Progressions - Two Pisier Type Problems
}

\author{
Dennis Davenport \\ Department of Mathematics \\ Miami University, Oxford, OH 45056, USA \\ davenpde@muohio.edu \\ Neil Hindman* \\ Department of Mathematics \\ Howard University, Washington, DC, 20059, USA \\ nhindman@aol.com \\ http://members.aol.com/nhindman/ \\ Dona Strauss \\ Department of Pure Mathematics \\ University of Hull, Hull HU6 7RX, UK \\ d.strauss@maths.hull.ac.uk
}

Submitted: May 31, 2001; Accepted: May 2, 2002.

MR Subject Classification: 05D10

\begin{abstract}
Let $\mathcal{P}_{f}(\mathbb{N})$ be the set of finite nonempty subsets of $\mathbb{N}$ and for $F, G \in \mathcal{P}_{f}(\mathbb{N})$ write $F<G$ when $\max F<\min G$. Let $X=\left\{(F, G): F, G \in \mathcal{P}_{f}(\mathbb{N})\right.$ and $\left.F<G\right\}$. A triangle in $X$ is a set of the form $\{(F \cup H, G),(F, G),(F, H \cup G)\}$ where $F<H<G$. Motivated by a question of Erdős, Nešetríl, and Rödl regarding three term arithmetic progressions, we show that any finite subset $Y$ of $X$ contains a relatively large triangle free subset. Exact values are obtained for the largest triangle free sets which can be guaranteed to exist in any set $Y \subseteq X$ with $n$ elements for all $n \leq 14$.
\end{abstract}

\footnotetext{
* This author acknowledges support received from the National Science Foundation (USA) via grant DMS-0070593.
} 


\section{Introduction.}

Our motivation for this study comes from a question of Erdős, Nešetríl, and Rödl [1, Problem 2, p. 221].

1.1 Question. Do there exist $\epsilon>0$ and a subset $X$ of $\mathbb{N}$ such that

(1) for every $r \in \mathbb{N}$, if $X=\bigcup_{i=1}^{r} C_{i}$, then there exist $i \in\{1,2, \ldots, r\}$ and $a, d \in \mathbb{N}$ with $\{a, a+d, a+2 d\} \subseteq C_{i}$ but

(2) for every finite subset $Y$ of $X$, there exists $Z \subseteq Y$ such that $|Z| \geq \epsilon \cdot|Y|$ and $Z$ does not contain any three term arithmetic progressions?

It is easy to see that Szemerédi's Theorem [8], or in fact only Roth's Theorem [6,7], implies that $X=\mathbb{N}$ does not satisfy (2) of Question 1.1.

Modifying a suggestion of Vitaly Bergelson, we came to consider "triangles" in the following set $X$. (Here $\mathcal{P}_{f}(\mathbb{N})=\{F \subseteq \mathbb{N}: F \neq \emptyset$ and $F$ is finite $\}$ and $F<G$ means that $\max F<\min G$.)

\subsection{Definition.}

(a) $X=\left\{(F, G): F, G \in \mathcal{P}_{f}(\mathbb{N})\right.$ and $\left.F<G\right\}$.

(b) A triangle in $X$ is a set of the form $\{(F \cup H, G),(F, G),(F, H \cup G)\}$ where $F, H, G \in$ $\mathcal{P}_{f}(\mathbb{N})$ and $F<H<G$.

We then address the following question.

1.3 Question. Does there exist $\epsilon>0$ such that, for any finite $Y \subseteq X$ there must exist $Z \subseteq Y$ such that $|Z| \geq \epsilon \cdot|Y|$ and $Z$ contains no triangles?

Questions 1.1 and 1.3 are both examples of what have come to be known as Pisier type problems. In [5], G. Pisier announced a proof that a subset $X$ of the dual group $\hat{G}$ of a compact abelian group $G$ is a Sidon set if and only if there is some $\epsilon>0$ such that for every finite subset $Y$ of $X$ there is a subset $Z$ of $Y$ such that $|Z| \geq \epsilon \cdot|Y|$ and $Z$ is quasi-independent, meaning that whenever $F$ and $G$ are distinct subsets of $Z$, $\sum F \neq \sum G$. See [1] and [2] for more information about Pisier type problems.

Our reason for asking Question 1.3 is contained in the following theorem.

1.4 Theorem. An affirmative answer to Question 1.3 implies an affirmative answer to Question 1.1.

Proof. Let $\left\langle x_{n}\right\rangle_{n=1}^{\infty}$ be a sequence in $\mathbb{N}$ with the property that for all $n \in \mathbb{N}, x_{n+1}>$ $4 \cdot \sum_{k=1}^{n} x_{k}$ and let

$$
X^{\prime}=\left\{\sum_{n \in G} x_{n}-\sum_{n \in F} x_{n}:(F, G) \in X\right\} .
$$

Let $r \in \mathbb{N}$ and let $X^{\prime}=\bigcup_{i=1}^{r} C_{i}$. For $i \in\{1,2, \ldots, r\}$, let

$$
\mathcal{D}_{i}=\left\{\{F, G\}: F, G \in \mathcal{P}_{f}(\mathbb{N}), F<G \text {, and }-\sum_{n \in F} x_{n}+\sum_{n \in G} x_{n} \in C_{i}\right\} .
$$

Pick by the Milliken-Taylor Theorem ([3, Theorem 2.2], [9, Lemma 2.2]) some $i \in\{1,2, \ldots, r\}$ and sets $F<H<G$ in $\mathcal{P}_{f}(\mathbb{N})$ such that

$$
\{(F \cup H, G),(F, G),(F, H \cup G)\} \subseteq \mathcal{D}_{i} .
$$


Let $a=-\sum_{n \in F \cup H} x_{n}+\sum_{n \in G} x_{n}$ and let $d=\sum_{n \in H} x_{n}$. As a consequence, part (1) of Question 1.1 holds for $X^{\prime}$.

To see that an affirmative answer to Question 1.3 implies part (2) of Question 1.1, it suffices to show that the only three term arithmetic progressions $\{a, a+d, a+2 d\}$ in $X^{\prime}$ are those with $a=\sum_{n \in G} x_{n}-\sum_{n \in F \cup H} x_{n}$ and $d=\sum_{n \in H} x_{n}$.

Assume that we have $a, d \in \mathbb{N}$ such that $\{a, a+d, a+2 d\} \subseteq X^{\prime}$. For $y \in X^{\prime}$ we can uniquely express $y$ as $\sum_{n=1}^{\infty} \pi_{n}(y) \cdot x_{n}$ where each $\pi_{n}(y) \in\{-1,0,1\}$. Further, if $\pi_{n}(y)=-1$ and $\pi_{m}(y)=1$, then $n<m$.

Let $b=a+d$ and $c=a+2 d$. Then

$$
\sum_{n=1}^{\infty}\left(\pi_{n}(b)-\pi_{n}(a)\right) \cdot x_{n}=b-a=d=c-b=\sum_{n=1}^{\infty}\left(\pi_{n}(c)-\pi_{n}(b)\right) \cdot x_{n} .
$$

It follows that $\pi_{n}(c)-\pi_{n}(b)=\pi_{n}(b)-\pi_{n}(a)$ for every $n \in \mathbb{N}$. Thus for each $n$, we have $\pi_{n}(a)=\pi_{n}(b)=\pi_{n}(c),\left(\pi_{n}(a), \pi_{n}(b), \pi_{n}(c)\right)=(-1,0,1)$, or $\left(\pi_{n}(a), \pi_{n}(b), \pi_{n}(c)\right)=$ $(1,0,-1)$. Since $d=b-a>0$, choose a largest $n$ such that $\pi_{n}(b)-\pi_{n}(a) \neq 0$ and note that $\pi_{n}(b)-\pi_{n}(a)>0$. Then $\left(\pi_{n}(a), \pi_{n}(b), \pi_{n}(c)\right)=(-1,0,1)$. If $m<n$, we can't have $\pi_{m}(a)=1$. Thus, for every $m$, either $\pi_{n}(a)=\pi_{n}(b)=\pi_{n}(c)$ or $\left(\pi_{n}(a), \pi_{n}(b), \pi_{n}(c)\right)=$ $(-1,0,1)$.

Our claim now follows with $F=\left\{n \in \mathbb{N}: \pi_{n}(b)=-1\right\}, G=\left\{n \in \mathbb{N}: \pi_{n}(b)=1\right\}$ and $H=\left\{n \in \mathbb{N} \backslash F: \pi_{n}(a)=-1\right\}$.

We are hopeful that the answer to Question 1.3 is "yes". And all of the evidence which we shall present later is consistent with an affirmative answer for $\epsilon=\frac{1}{2}$. However, we must point out that the referee does not share our optimism. One reason is that our set $X$ has "short cycles". For example, if

$$
\begin{aligned}
& T_{1}=\{(\{1,2\},\{4\}),(\{1\},\{4\}),(\{1\},\{2,4\})\}, \\
& T_{2}=\{(\{1,2,3\},\{4\}),(\{1\},\{4\}),(\{1\},\{2,3,4\})\}, \text { and } \\
& T_{3}=\{(\{1,2,3\},\{4\}),(\{1,2\},\{4\}),(\{1,2\},\{3,4\})\},
\end{aligned}
$$

Then $T_{1} \cap T_{2}=\{(\{1\},\{4\})\}, T_{2} \cap T_{3}=\{(\{1,2,3\},\{4\})\}$, and $T_{3} \cap T_{1}=\{(\{1,2\},\{4\})\}$. By way of contrast, given any $l \in \mathbb{N}$, Nešetríl and Rödl announced in [4] the existence of a set $S$ which satisfies part (1) of Question 1.1 but has no cycles of length at most $l$. (Recall that a cycle in a hypergraph consists of a finite sequence $E_{1}, E_{2}, \ldots, E_{n}$ of distinct edges such there exists a corresponding sequence $v_{1}, v_{2}, \ldots, v_{n}$ of distinct vertices with $v_{n} \in E_{1} \cap E_{n}$ and $v_{i} \in E_{i} \cap E_{i+1}$ for each $i \in\{1,2, \ldots, n-1\}$.) 


\section{2. "Triangles" among Pairs of Finite Sets}

We develop in this section some basic facts about triangles in $X$. Notice that triangles are uniquely represented. That is, if $\{(A, B),(C, D),(E, J)\}$ is a triangle in $X$ then there exist unique $(F, H, G) \in \mathcal{P}_{f}(\mathbb{N})^{3}$ with $F<H<G$ such that

$$
\{(F \cup H, G),(F, G),(F, H \cup G)\}=\{(A, B),(C, D),(E, J)\} .
$$

The following fact will also be useful.

2.1 Remark. Let $\mathcal{A}$ and $\mathcal{B}$ be distinct triangles in $X$. Then $|\mathcal{A} \cap \mathcal{B}| \leq 1$.

In the current context, we can turn Question 1.3 into a completely finite question.

2.2 Definition. Let $i, j \in \mathbb{N}$ with $i<j$. Then

$$
X_{i, j}=\{(F, G) \in X: \min F=i \text { and } \max G=j\} \text {. }
$$

2.3 Lemma. Any triangle in $X$ is contained in $X_{i, j}$ for some $i<j$.

Proof. Let $\{(F \cup H, G),(F, G),(F, H \cup G)\}$ be a triangle in $X$, let $i=\min F$ and let $j=\max G$.

2.4 Theorem. Let $\epsilon>0$. The following statements are equivalent.

(a) For every finite $Y \subseteq X$ there exists $Z \subseteq Y$ such that $|Z| \geq \epsilon \cdot|Y|$ and $Z$ contains no triangles.

(b) For all $i<j$ and every $Y \subseteq X_{i, j}$ there exists $Z \subseteq Y$ such that $|Z| \geq \epsilon \cdot|Y|$ and $Z$ contains no triangles.

Proof. That $(a)$ implies $(b)$ is trivial. To see that $(b)$ implies $(a)$, let $Y$ be a finite subset of $X$ and let $T=\left\{(i, j): Y \cap X_{i, j} \neq \emptyset\right\}$. For each $(i, j) \in T$, let $Y_{i, j}=Y \cap X_{i, j}$ and pick $Z_{i, j} \subseteq Y_{i, j}$ such that $\left|Z_{i, j}\right| \geq \epsilon \cdot X_{i, j}$ and $Z_{i, j}$ contains no triangles. Let $Z=\bigcup_{(i, j) \in T} Z_{i, j}$. Then $|Z| \geq \epsilon \cdot|Y|$ and, by Lemma 2.3, $Z$ contains no triangles.

We notice that the structure of $X_{i, j}$ depends only on $j-i$.

2.5 Remark. Let $k, i \in \mathbb{N}$. The function $\gamma: X_{1, k+1} \rightarrow X_{i, k+i}$ defined by $\gamma(F, G)=$ $(i-1+F, i-1+G)$ is a bijection which maps the set of triangles in $X_{1, k+1}$ onto the set of triangles in $X_{i, k+i}$.

An additional reduction is provided by the following lemma.

2.6 Lemma. Let $k \in \mathbb{N}$ and let $\epsilon>0$. The following statements are equivalent.

(a) For every $Y \subseteq X_{1, k+1}$ there exists $Z \subseteq Y$ such that $|Z| \geq \epsilon \cdot|Y|$ and $Z$ contains no triangles.

(b) For every $i \in \mathbb{N}$ and every $Y \subseteq X_{i, k+i}$ there exists $Z \subseteq Y$ such that $|Z| \geq \epsilon \cdot|Y|$ and $Z$ contains no triangles.

Proof. This is an immediate consequence of Remark 2.5.

We notice that there are large subsets of $X_{i, j}$ that have no triangles. 


\subsection{Definition.}

(a) For $F, G \in \mathcal{P}_{f}(\mathbb{N})$, let $\varphi(F, G)=\min G-\max F$.

(b) Let $i<j$ in $\mathbb{N}$ and let $t \in \omega$. Then $W_{i, j, t}=\left\{(F, G) \in X_{i, j}: 2^{t} \leq \varphi(F, G)<2^{t+1}\right\}$.

2.8 Lemma. Let $i<j$ in $\mathbb{N}$.

(a) Let $\{(F \cup H, G),(F, G),(F, H \cup G)\}$ be a triangle in $X_{i, j}$. Then $\varphi(F, G) \geq$ $\varphi(F \cup H, G)+\varphi(F, H \cup G)$.

(b) Let $t \in \omega$. Then $W_{i, j, t}$ contains no triangles.

Proof. Conclusion $(b)$ is immediate from conclusion $(a)$, which is trivial.

2.9 Lemma. Let $i<j$ in $\omega$ and let $k=j-i$.

(a) $\left|X_{i, j}\right|=(k+1) \cdot 2^{k-2}$.

(b) If $k \geq 2$, then $\left|W_{i, j, 0}\right|=(k+2) \cdot 2^{k-3}$.

Proof. (a) We proceed by induction on $k$. We know that $\left|X_{i, i+1}\right|=1$. Assume that $k=j-i \geq 1$ and $\left|X_{i, j}\right|=(k+1) \cdot 2^{k-2}$. Then

$$
\begin{aligned}
X_{i, j+1}= & \left\{(F,(G \backslash\{j\}) \cup\{j+1\}):(F, G) \in X_{i, j}\right\} \cup\left\{(F, G \cup\{j+1\}):(F, G) \in X_{i, j}\right\} \\
& \cup\{(\{i, j\} \cup H,\{j+1\}): H \subseteq\{i+1, i+2, \ldots, j-1\}\}
\end{aligned}
$$

and so $\left|X_{i, j+1}\right|=2 \cdot\left|X_{i, j}\right|+2^{k-1}=(k+1) \cdot 2^{k-1}+2^{k-1}=(k+2) 2^{k-1}$.

(b) Let $k=j-i \geq 2$. Then $W_{i, j, 0}=X_{i, j} \backslash\left\{(F, 1+G):(F, G) \in X_{i, j-1}\right\}$ and so $\left|W_{i, j, 0}\right|=\left|X_{i, j}\right|-\left|X_{i, j-1}\right|=(k+1) \cdot 2^{k-2}-k \cdot 2^{k-3}=(k+2) \cdot 2^{k-3}$.

Notice that, as a consequence of Lemmas 2.8 and 2.9, each $X_{i, j}$ has a subset which has no triangles and contains slightly more than half of the members of $X_{i, j}$.

We now introduce some numbers with which we shall be concerned for the rest of this paper.

\subsection{Definition.}

(a) For $Y \in \mathcal{P}_{f}(X), \mu(Y)=\max \{|Z|: Z \subseteq Y$ and $Z$ contains no triangles $\}$.

(b) For $k \in \mathbb{N}, \epsilon_{k}=\min \left\{\frac{\mu(Y)}{|Y|}: Y \in \mathcal{P}_{f}\left(X_{1, k+1}\right)\right\}$.

(c) For $k \in \mathbb{N}, \lambda_{k}=\min \left\{\mu(Y): Y \in \mathcal{P}_{f}(X)\right.$ and $\left.|Y|=k\right\}$.

We observe now that answering Question 1.3 amounts to determining bounds for the numbers $\lambda_{k}$ or $\epsilon_{k}$.

2.11 Lemma. Let $\epsilon>0$. The following statements are equivalent.

(a) For every finite $Y \subseteq X$ there exists $Z \subseteq Y$ such that $|Z| \geq \epsilon \cdot|Y|$ and $Z$ contains no triangles.

(b) For every $k \in \mathbb{N}, \lambda_{k} \geq \epsilon \cdot k$.

(c) For every $k \in \mathbb{N}, \epsilon_{k} \geq \epsilon$. 
Proof. To see that $(a)$ implies $(b)$, let $k \in \mathbb{N}$ and pick $Y \subseteq X$ such that $|Y|=k$ and $\mu(Y)=\lambda_{k}$. Pick $Z \subseteq Y$ such that $|Z| \geq \epsilon \cdot|Y|$ and $Z$ contains no triangles. Then $\lambda_{k}=\mu(Y) \geq|Z| \geq \epsilon \cdot k$.

To see that $(b)$ implies $(c)$, let $k \in \mathbb{N}$ and pick $Y \in \mathcal{P}_{f}\left(X_{1, k+1}\right)$ such that $\frac{\mu(Y)}{|Y|}=\epsilon_{k}$ and let $m=|Y|$. Then $\epsilon \cdot m \leq \lambda_{m} \leq \mu(Y)$ and so $\epsilon_{k} \geq \epsilon$.

That $(c)$ implies $(a)$ is an immediate consequence of Theorem 2.4 and Lemma 2.6.

2.12 Lemma. Let $k \in \mathbb{N}$.

(a) $\lambda_{k} \leq \lambda_{k+1} \leq \lambda_{k}+1$.

(b) $\epsilon_{k+1} \leq \epsilon_{k}$.

Proof. Conclusion $(a)$ is immediate. To verify conclusion $(b)$, observe that the function $\gamma: X_{1, k+1} \rightarrow X_{1, k+2}$ defined by $\gamma(F, G)=(F, G \cup\{k+2\})$ is injective and takes triangles to triangles.

\section{Values of $\lambda_{k}$}

In this section we derive exact values of $\lambda_{k}$ for $k \leq 13$ (and announce the value of $\lambda_{14}$ ). On the one hand, the fact that we can do this is somewhat surprising. (By way of contrast, we only know exact values of $\epsilon_{k}$ for $k \leq 4$.) On the other hand, the methods used are of an ad hoc nature and do not yield any lower bounds on the growth of $\lambda_{k}$. (Again by way of contrast, in the next section we shall establish reasonable lower bounds on the values of $\epsilon_{k}$, though not good enough to answer Question 1.3.)

We begin by recording some trivial values.

3.1 Theorem. $\lambda_{1}=1, \lambda_{2}=2, \lambda_{3}=2, \lambda_{4}=3, \lambda_{5}=4$, and $\lambda_{6}=4$.

Proof. The first three values are completely trivial, while the last three follow immediately from Remark 2.1 .

The notation introduced next does not indicate its dependence on the choice of $Y$. This should not be confusing as we will be mostly working with one $Y$ at a time.

3.2 Definition. Let $Y \subseteq X$ and let $x \in Y$. Then $C_{x}=\{y \in Y \backslash\{x\}$ : there exists a triangle $A$ in $Y$ with $\{x, y\} \subseteq A\}$ and $T_{x}=\mid\{A: A$ is a triangle in $Y$ and $x \in A\}$. We say that $T_{x}$ is the degree of $x$.

The following geometric notion will be helpful.

3.3 Definition. Let $Y \subseteq X$. Then $V$ and $W$ disconnect $Y$ if and only if $Y=V \cup W$, $V \cap W=\emptyset, V \neq \emptyset, W \neq \emptyset$, and any triangle which is contained in $Y$ is either contained in $V$ or contained in $W$. We say that $Y$ is disconnected if and only if there exist $V$ and $W$ that disconnect $Y$, and otherwise we say that $Y$ is connected.

3.4 Remark. Let $Y \subseteq X$ and let $V$ and $W$ disconnect $Y$. Then $\mu(Y)=\mu(V)+\mu(W)$. 
3.5 Lemma. Let $k, t \in \mathbb{N}$.

(a) $\lambda_{k+t} \leq \lambda_{k}+\lambda_{t}$.

(b) Let $Y \subseteq X$. If there exist $V$ and $W$ with $|V|=k$ and $|W|=t$ such that $V$ and $W$ disconnect $Y$, then $\mu(Y) \geq \lambda_{k}+\lambda_{t}$.

Proof. (a) Pick $V, W \subseteq X$ such that $|V|=k,|W|=t, \mu(V)=\lambda_{k}$, and $\mu(W)=\lambda_{t}$. By Remark 2.5 we may presume that $V$ and $W$ disconnect $V \cup W$, simply by translating $W$ sufficiently. Then $\lambda_{k+t} \leq \mu(V \cup W)=\mu(V)+\mu(W)$ by Remark 3.4.

(b) By Remark 3.4, $\mu(Y)=\mu(V)+\mu(W) \geq \lambda_{k}+\lambda_{t}$.

We shall find it convenient to have a linear ordering of $X$.

3.6 Definition. Given distinct $(F, G)$ and $\left(F^{\prime}, G^{\prime}\right)$ in $X,(F, G)<\left(F^{\prime}, G^{\prime}\right)$ if and only if $\max \left(\left(F^{\prime} \cup G\right) \triangle\left(F \cup G^{\prime}\right)\right) \in F \cup G^{\prime}$.

Notice that $(F, G)<\left(F^{\prime}, G^{\prime}\right)$ if and only if whenever $\left\langle x_{n}\right\rangle_{n=1}^{\infty}$ is a sequence with the property that $x_{n+1}>4 \cdot \sum_{k=1}^{n} x_{n}$ for each $n$, one has $\sum_{n \in F}-x_{n}+\sum_{n \in G} x_{n}<$ $\sum_{n \in F^{\prime}}-x_{n}+\sum_{n \in G^{\prime}} x_{n}$. In particular, given $F<H<G$, one has $(F \cup H, G)<$ $(F, G)<(F, H \cup G)$.

We now introduce coordinates for elements of $X$, defining an embedding of $X$ in $\{-1,0,1\}^{\mathbb{N}}$.

3.7 Definition. Let $x=(F, G) \in X$. For each $n \in \mathbb{N}$, we define

$$
\pi_{n}(x)=\left\{\begin{array}{cl}
-1 & \text { if } n \in F \\
0 & \text { if } n \notin F \cup G \\
1 & \text { if } n \in G .
\end{array}\right.
$$

We observe that, for any $x, y, z \in X$ with $x<y<z,\{x, y, z\}$ is a triangle if and only if, for every $n \in \mathbb{N}, \pi_{n}(x)=\pi_{n}(y)=\pi_{n}(z)$ or $\left(\pi_{n}(x), \pi_{n}(y), \pi_{n}(z)\right)=(-1,0,1)$.

3.8 Lemma. Let $x, y, z \in X$ be distinct, with $y<z$. Suppose that there are elements $p, q, r \in X$ for which $\{x, y, p\},\{x, z, q\}$ and $\{y, z, r\}$ are triangles and $\{x, y, p\} \neq$ $\{x, z, q\}$. Then one of the following nine cases holds.

(a) $x<y<p, x<z<q$, and $y<z<r$

(b) $y<x<p, x<z<q$, and $y<z<r$

(c) $y<x<p, z<x<q$, and $y<z<r$

(a) $x<p<y, x<q<z$, and $y<r<z$

(II) (b) $y<p<x, x<q<z$, and $y<r<z$

(c) $y<p<x, z<q<x$, and $y<r<z$

(a) $p<y<x, q<z<x$, and $r<y<z$

(III) (b) $p<y<x, q<x<z$, and $r<y<z$

(c) $p<x<y, q<x<z$, and $r<y<z$. 
Proof. In the following array, $\{a, b, c\}=\{-1,0,1\}=\{u, v, w\}$. Each column gives possible values of $\left(\pi_{n}(x), \pi_{n}(y), \pi_{n}(z), \pi_{n}(p), \pi_{n}(q), \pi_{n}(r)\right)$ for some value of $n$. The first three columns display all non-constant possibilities with $\pi_{n}(y) \neq \pi_{n}(z)$ and the last displays the only non-constant possibility with $\pi_{n}(y)=\pi_{n}(z)$. (Notice that, since $\{y, z, r\}$ is a triangle, if $n, m \in \mathbb{N}, \pi_{n}(y) \neq \pi_{n}(z)$, and $\pi_{m}(y) \neq \pi_{m}(z)$, then $\left(\pi_{n}(y), \pi_{n}(z)\right)=\left(\pi_{m}(y), \pi_{m}(z)\right)$. If, for example, $y<z<r$, this common value must be $(-1,0)$.)

$\begin{array}{llllll}x & - & a & b & c & u \\ y & - & b & b & b & v \\ z & - & c & c & c & v \\ p & - & c & b & a & w \\ q & - & b & a & c & w \\ r & - & a & a & v\end{array}$

We make some observations. At least two of these four columns must occur for some $n$. (If the first is the only one which occurs, then $y=q$ and $z=p$ and so $\{x, y, p\}=\{x, z, q\}$. If the second is the only one which occurs, then $x=y$; if the third is the only one which occurs, then $x=z$; if the fourth is the only one which occurs, then $y=z$.) On the other hand, we claim that the first column cannot occur with any of the others. If the first and second columns both occur, then $a<b$ and the fact that $\{x, z, q\}$ is a triangle implies that $x<q$ and $q<x$, while $b<a$ implies that $q<x$ and $x<q$. Similarly, the occurence of the first and third columns leads to a contradiction. If the first and fourth columns both occur, then the fact that $\{x, y, p\}$ is a triangle implies that $u=a, v=b$ and $w=c$. Since $y<z$, it follows that $b<c$, and this implies that $q<z$ and $z<q$. So the first column cannot occur.

We can now prove the lemma.

We obtain case I by choosing $a=1, b=-1$, and $c=0$. If columns 2 and 3 both occur, we have $\mathrm{I}(\mathrm{b})$. If columns 2 and 4 both occur, then the fact that $\{x, z, q\}$ is a triangle implies that $u=b=-1, v=c=0$, and $w=a=1$. This gives us I(a). If columns 3 and 4 both occur, we obtain I(c).

In a similar way, we obtain case II by choosing $a=0, b=-1$, and $c=1$. We obtain case III by choosing $a=-1, b=0$, and $c=1$.

This exhausts the possibilities, because the assumption that $y<z$ implies that $b<c$.

The following consequence of the above lemma will frequently be useful.

3.9 Theorem. Let $Y \subseteq X$ and let $x \in Y$. Then $C_{x}$ contains no triangles.

Proof. Suppose that $\{w, y, z\}$ is a triangle contained in $C_{x}$. We may suppose that $w<y<z$. Let $p, q, r$ be elements of $Y$ for which $\{x, y, p\},\{x, z, q\}$ and $\{x, w, r\}$ are triangles. We claim that $\{x, y, p\} \neq\{x, z, q\}$. Suppose instead that $\{x, y, p\}=\{x, z, q\}$. Then $(y, p)=(q, z)$ and so $\{x, y, p\}=\{x, y, z\}$. Thus by Remark $2.1\{x, y, z\}=$ $\{w, y, z\}$, and so $w=x$, contradicting the fact that $w \in C_{x}$. Similarly one may show that $\{x, z, q\} \neq\{x, w, r\}$. 
We can apply Lemma 3.8 with $r$ replaced by $w$, and see that one of the possibilities listed in III must hold. So $q<z<x$ or $q<x<z$.

We can also apply Lemma 3.8 with $y, p, r$ replaced by $w, r, y$ respectively. Since $q<x<z$ or $q<z<x$, III must still hold. However, each of the three possibilities listed in III now implies that $y<w$, a contradiction.

3.10 Theorem. $\lambda_{7}=5$.

Proof. By Lemma 2.12 and Theorem 3.1, $\lambda_{7} \leq 5$. To see that $\lambda_{7} \geq 5$, let $Y \subseteq X$ with $|Y|=7$. Assume first that there is some $y$ with $T_{y}=3$. Then $C_{y}=Y \backslash\{y\}$ and by Theorem $3.9 C_{y}$ is triangle free.

Thus we may assume that for each $y \in Y, T_{y} \leq 2$, and consequently there are at most 4 triangles in $Y$. (If there were 5 triangles in $Y$, we would have $\sum_{y \in Y} T_{y} \geq 15$.) If there are 3 or fewer, the result is immediate, so suppose that $A, B, C$, and $D$ are distinct triangles in $Y$. If some pair is disjoint, we may assume that $A \cap B=\emptyset$. But then $A \cap C \neq \emptyset$ and $B \cap D \neq \emptyset$. So, in any event, we may pick $y \in A \cap C$ and $z \in B \cap D$. Then $Y \backslash\{y, z\}$ is triangle free.

For the remainder of this section we turn our attention to establishing the values of $\lambda_{8}$ and $\lambda_{12}$ (from which the values for $\lambda_{9}, \lambda_{10}, \lambda_{11}$, and $\lambda_{13}$ follow immediately). The major tool for this effort is the known structure of $X_{1,4}$, which we pause now to describe in some detail. (It is surprising that the structure of $X_{1,4}$, which has only eight elements, allows us to deduce the exact values of $\lambda_{12}$ and $\lambda_{13}$. In fact, in a proof that we will not present, it gives us significant information even about $\lambda_{26}$.)

3.11 Definition. $S=\{-1,0,1\}^{2} \backslash\{(1,-1)\}$.

We can identify $S$ with $X_{1,4}$ by using the mapping $x \mapsto\left(\pi_{2}(x), \pi_{3}(x)\right)$ from $X_{1,4}$ onto $S$. Thus we shall regard $S$ as containing triangles.

We enumerate the elements of $S$ and the triangles in $S$ as follows:

$$
\begin{aligned}
& s_{1}=(1,1) \quad s_{2}=(0,0) \quad s_{3}=(-1,-1) \quad s_{4}=(0,1) \\
& s_{5}=(-1,1) \quad s_{6}=(-1,0) \quad s_{7}=(0,-1) \quad s_{8}=(1,0) \\
& V_{1}=\left\{s_{1}, s_{2}, s_{3}\right\} \quad V_{2}=\left\{s_{1}, s_{4}, s_{5}\right\} \quad V_{3}=\left\{s_{2}, s_{4}, s_{7}\right\} \\
& V_{4}=\left\{s_{2}, s_{6}, s_{8}\right\} \quad V_{5}=\left\{s_{3}, s_{5}, s_{6}\right\}
\end{aligned}
$$

Notice that $V_{1}, V_{2}, V_{3}, V_{4}$, and $V_{5}$ are exactly the triangles in $S$. Also, note that $\left\{s_{1}, s_{3}, s_{4}, s_{6}, s_{7}, s_{8}\right\}$ is a triangle free subset of $S$. The reader may find the following diagram helpful in following some of the arguments. 


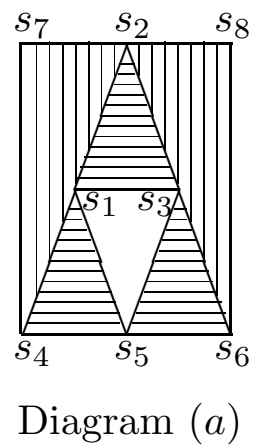

In the above diagram, the five triangles in $S$ are indicated by the shaded regions. (So, even though $s_{1}, s_{3}$, and $s_{5}$ are vertices of a geometric triangle in this diagram, $\left\{s_{1}, s_{3}, s_{5}\right\}$ is not a triangle in $S$.)

3.12 Definition. Let $Y$ and $W$ be sets, each with specified sets of "triangles", i.e. three element subsets. A function $\sigma: Y \rightarrow W$ is a triangle map if and only if for each triangle $A \subseteq Y$, either $|\sigma[A]|=1$ or $\sigma[A]$ is a triangle in $W$.

Notice that the composition of triangle maps is again a triangle map.

3.13 Remark. Let $Y \subseteq X$ and let $\sigma: Y \rightarrow X$ be a triangle map. If $Y$ is connected, then $\sigma[Y]$ is connected.

We shall use triangle maps which are created by one particular method.

3.14 Lemma. Let $Y \subseteq X$ with $|Y| \geq 5$ and assume that $T_{y} \leq 1$ for at most one $y \in Y$. Then there is a triangle map $\sigma: Y \rightarrow S$ such that $\left\{s_{1}, s_{2}, s_{3}, s_{4}, s_{5}\right\} \subseteq \sigma[Y]$.

Proof. Let $y=\max Y$ and let $z=\min Y$. Assume first that $T_{y} \geq 2$. Then there are distinct triangles $\left\{y, y_{1}, y_{2}\right\}$ and $\left\{y, y_{3}, y_{4}\right\}$ in $Y$. We may suppose that $y_{1}>y_{2}$ and $y_{3}>y_{4}$. For any $n \in \mathbb{N},\left(\left(\pi_{n}(y), \pi_{n}\left(y_{1}\right), \pi_{n}\left(y_{2}\right), \pi_{n}\left(y_{3}\right), \pi_{n}\left(y_{4}\right)\right)\right.$ is either constant or one of the following vectors: $(1,0,-1,0,-1) ;(1,0,-1,1,1)$; or $(1,1,1,0,-1)$.

Since the elements $y, y_{1}, y_{2}, y_{3}, y_{4}$ are distinct, at least two of these possibilities must occur. The second and third cannot both occur, since for any $n<m$ in $\mathbb{N}$ and any $w \in X$, one cannot have $\pi_{n}(w)=1$ and $\pi_{m}(w)=-1$. We may assume that the second occurs, since this could be achieved, if necessary, by interchanging the order of our two triangles. So there exist $m<n$ in $\mathbb{N}$ for which

$$
\begin{aligned}
\left(\left(\pi_{m}(y), \pi_{m}\left(y_{1}\right), \pi_{m}\left(y_{2}\right), \pi_{m}\left(y_{3}\right), \pi_{m}\left(y_{4}\right)\right)\right. & =(1,0,-1,0,-1) \text { and } \\
\left(\left(\pi_{n}(y), \pi_{n}\left(y_{1}\right), \pi_{n}\left(y_{2}\right), \pi_{n}\left(y_{3}\right), \pi_{n}\left(y_{4}\right)\right)\right. & =(1,0,-1,1,1) .
\end{aligned}
$$

We can now define the required triangle map $\sigma: Y \rightarrow S$ by $\sigma(x)=\left(\pi_{m}(x), \pi_{n}(x)\right)$.

If $T_{z} \geq 2$, we can use a similar argument to define a triangle map from $Y$ to $S$ which has $s_{1}, s_{2}, s_{3}, s_{5}, s_{6}$ in its image. To define $\sigma$, we follow this map by the triangle map from $S$ to itself obtained by interchanging -1 and 1 and reversing the order of the coordinates.

The following simple fact will frequently be useful. 
3.15 Lemma. Let $Y \subseteq X$, let $\sigma: Y \rightarrow X$ be a triangle map, and let $U=$ $\left\{t \in \sigma[Y]:\left|\sigma^{-1}[\{t\}]\right|=1\right\}$. Assume that $B$ is a triangle free subset of $\sigma[Y]$. Then $\mu(Y) \geq|B \cap U|+2 \cdot|B \backslash U|$.

Proof. For each $t \in B \cap U$, pick $x_{t} \in Y$ such that $\sigma\left(x_{t}\right)=t$. For each $t \in B \backslash U$, pick $y_{t} \neq z_{t}$ in $Y$ such that $\sigma\left(y_{t}\right)=\sigma\left(z_{t}\right)=t$. Let $Z=\left\{x_{t}: t \in B \cap U\right\} \cup\left\{y_{t}: t \in\right.$ $B \backslash U\} \cup\left\{z_{t}: t \in B \backslash U\right\}$. Since $\sigma$ does not take any three points in $Z$ to the same point, any triangle in $Z$ would have to go to a triangle in $B$.

3.16 Lemma. Let $Y \subseteq X$ with $|Y|=8$ and suppose that $\mu(Y) \leq 5$. Then $Y$ contains exactly 5 triangles and $\left|\left\{y \in Y: T_{y}=2\right\}\right|=7$ and $\left|\left\{y \in Y: T_{y}=1\right\}\right|=1$.

Proof. Since $|Y|=8$ we have immediately that for all $y \in Y, T_{y} \leq 3$. Suppose we have some $y$ with $T_{y}=3$. Then $\left|C_{y}\right|=6$ while by Theorem $3.9 C_{y}$ contains no triangles. Thus we must have that for all $y \in Y, T_{y} \leq 2$ and thus $\sum_{y \in Y} T_{y} \leq 16$. Consequently, $Y$ contains at most 5 triangles and trivially $Y$ contains at least 4 triangles.

If $Y$ contained only four triangles, then by a routine case analysis we could find triangles $A, B, C$, and $D$ with $A \cap B \neq \emptyset$ and $C \cap D \neq \emptyset$. Picking $y \in A \cap B$ and $z \in C \cap D$, one has that $Y \backslash\{y, z\}$ contains no triangles.

Thus $Y$ contains exactly 5 triangles and consequently $\sum_{y \in Y} T_{y}=15$.

3.17 Lemma. Let $Y$ be a connected subset of $S$.

(a) If $|Y|=5$, then there exist distinct $t_{1}, t_{2}, t_{3}, t_{4}$, and $t_{5}$ such that the triangles contained in $Y$ are exactly $\left\{t_{1}, t_{2}, t_{3}\right\}$ and $\left\{t_{1}, t_{4}, t_{5}\right\}$.

(b) If $|Y|=6$, then there exist distinct $t_{1}, t_{2}, t_{3}, t_{4}, t_{5}$, and $t_{6}$ such that the triangles contained in $Y$ are exactly $\left\{t_{1}, t_{2}, t_{3}\right\},\left\{t_{1}, t_{4}, t_{5}\right\}$, and $\left\{t_{3}, t_{4}, t_{6}\right\}$.

Proof. Conclusion $(a)$ is immediate. To verify conclusion $(b)$, note that since $Y$ is connected, it must contain at least three triangles and since $|Y|=6$, there can be no point of degree 3. Any two points of $S$ lie in at least two distinct triangles, and thus $Y$ contains exactly 3 triangles. Two disjoint triangles in a six element set do not leave room for a third, so no two are disjoint.

Lemma 3.17 says that the possible configurations for a five or six element connected subset of $S$ are as diagrammed below.

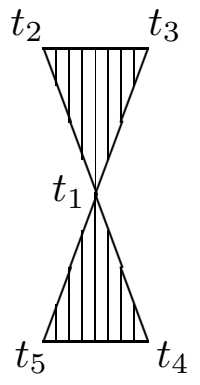

Diagram $(b)$

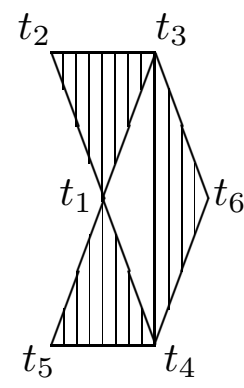

Diagram $(c)$

3.18 Theorem. $\lambda_{8}=6$. 
Proof. Suppose that we have $Y \subseteq X$ with $|Y|=8$ such that $\mu(Y) \leq 5$. Pick by Lemmas 3.14 and 3.16 a triangle map $\sigma: Y \rightarrow S$ such that $\left\{s_{1}, s_{2}, s_{3}, s_{4}, s_{5}\right\} \subseteq \sigma[Y]$.

Since $\left\{s_{1}, s_{3}, s_{4}, s_{6}, s_{7}, s_{8}\right\}$ is triangle free, we have that $\left\{s_{1}, s_{3}, s_{4}, s_{6}, s_{7}, s_{8}\right\} \backslash \sigma[Y] \neq$ $\emptyset$. (Otherwise, picking $y_{i} \in \sigma^{-1}\left[\left\{s_{i}\right\}\right]$ for each $i \in\{1,3,4,6,7,8\}$ would yield a six element triangle free subset of $Y$.) Since $\left\{s_{1}, s_{3}, s_{4}, s_{6}, s_{7}, s_{8}\right\} \subseteq \bigcup_{i=1}^{5} V_{i}$, there must be some $i$ such that $V_{i} \backslash \sigma[Y] \neq \emptyset$.

Since $\sigma$ is a triangle map and $Y$ contains 5 triangles by Lemma 3.16, either some triangle $A \subseteq Y$ has $|\sigma[A]|=1$ or some distinct triangles $A$ and $B$ in $Y$ have $\sigma[A]=\sigma[B]$. In either case we have $|\sigma[Y]| \leq 6$.

Notice also that $Y$ is connected. (This follows from Lemma 3.5 and the fact that $\lambda_{1}+\lambda_{7}=\lambda_{2}+\lambda_{6}=\lambda_{3}+\lambda_{5}=\lambda_{4}+\lambda_{4}=6$.) Hence, $\sigma[Y]$ is also. Thus by Lemma 3.17 we have that either

(a) there exist distinct $t_{1}, t_{2}, t_{3}, t_{4}$, and $t_{5}$ such that the triangles contained in $\sigma[Y]$ are exactly $\left\{t_{1}, t_{2}, t_{3}\right\}$ and $\left\{t_{1}, t_{4}, t_{5}\right\}$ or

(b) there exist distinct $t_{1}, t_{2}, t_{3}, t_{4}, t_{5}$, and $t_{6}$ such that the triangles contained in $\sigma[Y]$ are exactly $\left\{t_{1}, t_{2}, t_{3}\right\},\left\{t_{1}, t_{4}, t_{5}\right\}$, and $\left\{t_{3}, t_{4}, t_{6}\right\}$. (See diagrams $(b)$ and $(c)$ above.)

Let $U=\left\{x \in \sigma[Y]: \mid \sigma^{-1}[\{x\}]=1\right\}$. Assume first that (a) holds. By Lemma 3.15, $\left|\left\{t_{2}, t_{3}, t_{4}, t_{5}\right\} \cap U\right| \geq 3$. Assume without loss of generality that $\left\{t_{2}, t_{3}\right\} \subseteq U$. Pick (the unique) $y_{2}$ and $y_{3}$ in $Y$ such that $\sigma\left(y_{2}\right)=t_{2}$ and $\sigma\left(y_{3}\right)=t_{3}$. Since $y_{2} \neq y_{3}$ we may assume by Lemma 3.16 that $T_{y_{2}}=2$. Pick distinct triangles $A$ and $B$ in $Y$ such that $y_{2} \in A \cap B$ (so that $\left\{y_{2}\right\}=A \cap B$ ). Then $t_{2} \in U \cap \sigma[A] \cap \sigma[B]$, so $|\sigma[A]|>1$ and $|\sigma[B]|>1$ and hence $\sigma[A]=\sigma[B]=\left\{t_{1}, t_{2}, t_{3}\right\}$. But then $t_{3} \notin U$, a contradiction.

Now assume that $(b)$ holds. By Lemma 3.15, $\left\{t_{2}, t_{5}, t_{6}\right\} \cap U \neq \emptyset$. Without loss of generality, $t_{5} \in U$. We claim that $\left\{t_{1}, t_{2}\right\} \backslash U \neq \emptyset$. Suppose instead that $\left\{t_{1}, t_{2}\right\} \subseteq U$. Let $Y_{1}=\sigma^{-1}\left[\left\{t_{2}, t_{5}\right\}\right]$ and let $Y_{2}=\sigma^{-1}\left[\left\{t_{3}, t_{4}, t_{6}\right\}\right]$. Then $\left|Y_{1}\right|=2$ and (since $t_{1} \in U$ ) $\left|Y_{2}\right|=5$. If $Y_{1}$ and $Y_{2}$ disconnect $Y_{1} \cup Y_{2}$, we have by Lemma 3.5(b) that $\mu(Y) \geq$ $\mu\left(Y_{1} \cup Y_{2}\right) \geq \lambda_{2}+\lambda_{5}=6$. So there is some triangle $A \subseteq Y_{1} \cup Y_{2}$ such that $A \cap Y_{1} \neq \emptyset$ and $A \cap Y_{2} \neq \emptyset$. But then $\sigma[A]$ is a triangle and $t_{1} \notin \sigma[A]$ so $\sigma[A]=\left\{t_{3}, t_{4}, t_{6}\right\}$, a contradiction.

Similarly $\left\{t_{4}, t_{6}\right\} \backslash U \neq \emptyset$. But then $\left|\left\{t_{1}, t_{2}, t_{4}, t_{6}\right\} \backslash U\right| \geq 2$ so that, by Lemma 3.15 , $\mu(Y) \geq 6$.

3.19 Corollary. $\lambda_{9}=\lambda_{10}=6$.

Proof. By Lemma 2.12 and Theorem 3.18, $\lambda_{10} \geq \lambda_{9} \geq \lambda_{8}=6$.

To see that $\lambda_{10} \leq 6$ we produce a 10 element subset of $X_{1,5}$ with $\mu(Y)=6$. Let $Y=\left\{x_{1}, x_{2}, \ldots, x_{10}\right\}$, where

$$
\begin{array}{lll}
x_{1}=(\{1\},\{5\}) & x_{2}=(\{1,2\},\{5\}) & x_{3}=(\{1\},\{4,5\}) \\
x_{4}=(\{1,2,4\},\{5\}) & x_{5}=(\{1,2\},\{4,5\}) & x_{6}=(\{1\},\{2,4,5\}) \\
x_{7}=(\{1,2,3,4\},\{5\}) & x_{8}=(\{1,2,3\},\{4,5\}) & x_{9}=(\{1,2\},\{3,4,5\}) \\
x_{10}=(\{1\},\{2,3,4,5\}) & &
\end{array}
$$


Suppose that we have $Z \subseteq Y$ such that $|Z|=7$ and $Z$ contains no triangles. Suppose first that $x_{5} \in Z$. Now $\left\{x_{2}, x_{4}, x_{5}\right\},\left\{x_{3}, x_{5}, x_{6}\right\}$, and $\left\{x_{5}, x_{8}, x_{9}\right\}$ are all triangles. So at most one each of $\left\{x_{2}, x_{4}\right\},\left\{x_{3}, x_{6}\right\}$, and $\left\{x_{8}, x_{9}\right\}$ are in $Z$. Since that eliminates 3 elements from $Y$, we have the triangle $\left\{x_{1}, x_{7}, x_{10}\right\} \subseteq Z$, a contradiction. Thus $x_{5} \notin Z$.

Now suppose that $x_{1} \in Z$. Since $\left\{x_{1}, x_{4}, x_{6}\right\}$ and $\left\{x_{1}, x_{7}, x_{10}\right\}$ are triangles, at most one each of $\left\{x_{4}, x_{6}\right\}$ and $\left\{x_{7}, x_{10}\right\}$ are in $Z$. So, since $x_{5} \notin Z$, we must have $\left\{x_{1}, x_{2}, x_{3}, x_{8}, x_{9}\right\} \subseteq Z$. Now $\left\{x_{2}, x_{7}, x_{9}\right\}$ is a triangle so $x_{7} \notin Z$. Also $\left\{x_{3}, x_{8}, x_{10}\right\}$ is a triangle so $x_{10} \notin Z$. But now we have eliminated $x_{5}, x_{7}, x_{10}$, and one of $\left\{x_{4}, x_{6}\right\}$ from $Z$. Thus $x_{1} \notin Z$.

But now $\left\{x_{2}, x_{7}, x_{9}\right\}$ and $\left\{x_{3}, x_{8}, x_{10}\right\}$ are disjoint triangles, so two more elements are eliminated from $Z$.

Now we turn our attention to $\lambda_{12}$.

3.20 Lemma. Let $Y \subseteq X$ with $|Y|=11$. if either

(i) there are four points $z_{1}, z_{2}, z_{3}$, and $z_{4}$ in $Y$ with $T_{y_{i}}=1$ for each $i$ or

(ii) there exist two points $y$ and $z$ in $Y$ with $T_{y}=0$ and $T_{z} \leq 1$, then $\mu(Y)=8$.

Proof. $(i)$ For $i \in\{1,2,3,4\}$ let $A_{i}$ be the unique triangle including $z_{i}$. Since $|Y|<12$, there exist $i \neq j$ such that $A_{i} \cap A_{j} \neq \emptyset$. (Possibly $A_{i}=A_{j}$.) Pick $x \in\left(A_{i} \cap A_{j}\right) \backslash\left\{z_{i}, z_{j}\right\}$. Since $\lambda_{8}=6$, pick a six point triangle free set $Z \subseteq Y \backslash\left\{x, z_{i}, z_{j}\right\}$. Then $Z \cup\left\{z_{i}, z_{j}\right\}$ is triangle free.

(ii) Choose $x \in Y \backslash\{y, z\}$ with $x \in C_{z}$ if $T_{z}=1$. Choose a six point triangle free set $Z \subseteq Y \backslash\{x, y, z\}$. Then $Z \cup\{y, z\}$ is triangle free.

3.21 Lemma. Let $Y \subseteq X$ with $|Y|=12$ and assume that $\mu(Y) \leq 7$.

(a) There is at most one point $y \in Y$ with $T_{y} \leq 1$.

(b) If $D \subseteq Y,|D| \geq 11$, and $V$ and $W$ disconnect $D$, then $|V|=1$ or $|W|=1$.

Proof. (a) Suppose $y$ and $z$ are distinct points of $Y$ with $T_{y} \leq 1$ and $T_{z} \leq 1$. Pick $u \in Y \backslash\{y\}$ and $v \in Y \backslash\{z\}$ with $u \in C_{y}$ if $T_{y}=1$ and with $v \in C_{z}$ if $T_{z}=1$. Then $Y \backslash\{u, v, y, z\}$ has eight or nine points so pick a six point triangle free set $Z \subseteq$ $Y \backslash\{u, v, y, z\}$. Then $Z \cup\{y, z\}$ is triangle free.

(b) Let $k=|V|$ and $t=|W|$. By Lemma 3.5, $\mu(D) \geq \lambda_{k}+\lambda_{t}$. And $\lambda_{2}+\lambda_{9}=$ $\lambda_{3}+\lambda_{8}=\lambda_{4}+\lambda_{7}=\lambda_{5}+\lambda_{6}=8$.

For the proof of the following lemma it may be helpful to refer to Diagram $(a)$.

3.22 Lemma. Let $Y \subseteq X$ with $|Y|=12$ and assume that $\mu(Y) \leq 7$ and $\sigma: Y \rightarrow S$ is a triangle-map. Then $|\sigma[Y]| \leq 7$ and, if $|\sigma[Y]|=7$, then $\left\{s_{6}, s_{7}, s_{8}\right\} \subseteq \sigma[Y]$.

Proof. Suppose first that $\sigma[Y]=S$. Let $U=\left\{s \in \sigma[Y]:\left|\sigma^{-1}[\{s\}]\right|=1\right\}$. By Lemma $3.15,\left|\left\{s_{1}, s_{3}, s_{4}, s_{6}, s_{7}, s_{8}\right\} \cap U\right| \geq 5$. Now

$$
Y \backslash \sigma^{-1}\left[\left\{s_{4}\right\}\right]=\sigma^{-1}\left[\left\{s_{7}\right\}\right] \cup \sigma^{-1}\left[\left\{s_{1}, s_{2}, s_{3}, s_{5}, s_{6}, s_{8}\right\}\right]
$$


so by Lemma 3.21(b), if $s_{4} \in U$, then also $s_{7} \in U$. Since one of these must by in $U$ we have in fact that $s_{7} \in U$. By symmetry $s_{8} \in U$.

Assume that $\left\{s_{4}, s_{6}\right\} \subseteq U$. Pick $Z \subseteq Y \backslash \sigma^{-1}\left[\left\{s_{4}, s_{6}, s_{7}, s_{8}\right\}\right]$ such that $Z$ has six elements and is triangle free. Then $Z \cup \sigma^{-1}\left[\left\{s_{7}, s_{8}\right\}\right]$ is triangle free. Thus we have that either $s_{4} \notin U$ or $s_{6} \notin U$. We assume without loss of generality that $s_{4} \notin U$. Then $\left\{s_{1}, s_{3}, s_{6}\right\} \subseteq U$. Pick a six point triangle free set $Z \subseteq Y \backslash \sigma^{-1}\left[\left\{s_{1}, s_{3}, s_{6}, s_{8}\right\}\right]$. Then $Z \cup \sigma^{-1}\left[\left\{s_{3}, s_{8}\right\}\right]$ is triangle free.

Thus we have established that $|\sigma[Y]| \leq 7$. Assume now that $|\sigma[Y]|=7$. Suppose that $s_{7} \notin \sigma[Y]$. Then by Lemma 3.15, $\left|\left\{s_{1}, s_{3}, s_{4}, s_{6}, s_{8}\right\} \backslash U\right| \leq 2$. Now $Y \backslash \sigma^{-1}\left[\left\{s_{3}\right\}\right]=$ $\sigma^{-1}\left[\left\{s_{2}, s_{6}, s_{8}\right\}\right] \cup \sigma^{-1}\left[\left\{s_{1}, s_{4}, s_{5}\right\}\right]$. Thus, by Lemma $3.21(b),\left|Y \backslash \sigma^{-1}\left[\left\{s_{3}\right\}\right]\right| \neq 11$ so that $s_{3} \notin U$. Now $Y \backslash \sigma^{-1}\left[\left\{s_{1}\right\}\right]=\sigma^{-1}\left[\left\{s_{4}\right\}\right] \cup \sigma^{-1}\left[\left\{s_{2}, s_{3}, s_{5}, s_{6}, s_{8}\right\}\right]$ so, again by Lemma $3.21(b)$, if $s_{1} \in U$, then $s_{4} \in U$. Also $Y \backslash \sigma^{-1}\left[\left\{s_{6}\right\}\right]=\sigma^{-1}\left[\left\{s_{8}\right\}\right] \cup \sigma^{-1}\left[\left\{s_{1}, s_{2}, s_{3}, s_{4}, s_{5}\right\}\right]$ so, if $s_{6} \in U$, then $s_{8} \in U$. Since $\left|\left\{s_{1}, s_{4}, s_{6}, s_{8}\right\} \backslash U\right| \leq 1$, we have then that $\left\{s_{4}, s_{8}\right\} \subseteq U$ and either $s_{1} \in U$ or $s_{6} \in U$.

The map from $S \backslash\left\{s_{7}\right\}$ to itself which sends $s_{3}$ to $s_{3}$ and interchanges the pairs $\left(s_{1}, s_{6}\right),\left(s_{4}, s_{8}\right)$, and $\left(s_{2}, s_{5}\right)$ is a triangle map, so we may assume without loss of generality that $s_{1} \in U$. If $y_{4} \in Y$ satisfies $s\left(y_{4}\right)=s_{4}$, then $y_{4}$ has degree 0 or 1 . Otherwise, if $y_{4}$ belonged to two distinct triangles, these would both be mapped to $\left\{s_{1}, s_{4}, s_{5}\right\}$ by $\sigma$ and this would imply that $s_{1} \notin U$. Hence, by Lemma 3.21(a), the unique point in $\sigma^{-1}\left[\left\{s_{8}\right\}\right]$ has degree at least 2 , and so $s_{2}, s_{6} \notin U$. We have already seen that $s_{3} \notin U$. It now follows from Lemma 3.15 and the fact that $\left\{s_{2}, s_{3}, s_{4}, s_{5}, s_{8}\right\}$ is triangle free, that $s_{5} \in U$.

We claim that $\left|\sigma^{-1}\left[\left\{s_{2}\right\}\right]\right| \geq 3$. Suppose instead that $\left|\sigma^{-1}\left[\left\{s_{2}\right\}\right]\right|=2$. Choose a six point triangle free subset $Z$ of $Y \backslash \sigma^{-1}\left[\left\{s_{1}, s_{2}, s_{8}\right\}\right]$. Then $\sigma^{-1}\left[\left\{s_{2}\right\}\right] \cup Z$ is an eight point triangle free set. Similarly, if $\left|\sigma^{-1}\left[\left\{s_{3}\right\}\right]\right|=2$, then the union of a six point triangle free subset of $Y \backslash \sigma^{-1}\left[\left\{s_{1}, s_{3}, s_{5}\right\}\right]$ with $\sigma^{-1}\left[\left\{s_{3}\right\}\right]$ is an eight point triangle free set. And if $\left|\sigma^{-1}\left[\left\{s_{6}\right\}\right]\right|=2$, then the union of a six point triangle free subset of $Y \backslash \sigma^{-1}\left[\left\{s_{5}, s_{6}, s_{8}\right\}\right]$ with $\sigma^{-1}\left[\left\{s_{6}\right\}\right]$ is an eight point triangle free set. But then $|Y| \geq 13$.

Thus we have established that $s_{7} \in \sigma[Y]$. By symmetry, $s_{8} \in \sigma[Y]$. To complete the proof, we suppose that $s_{6} \notin \sigma[Y]$. Then by Lemma 3.21, $s_{1}, s_{2}, s_{4} \notin U$, because, for each $i \in\{1,2,4\}, \sigma[Y] \backslash\left\{s_{i}\right\}$ is the disconnected union of two sets, neither of which is a singleton. Since $\left\{s_{1}, s_{3}, s_{8}, s_{7}, s_{4}\right\}$ is triangle free, it follows from Lemma 3.15 that $\left\{s_{3}, s_{7}, s_{8}\right\} \subseteq U$. Since $\left\{s_{5}, s_{1}, s_{2}, s_{7}, s_{8}\right\}$ is triangle free, it also follows from Lemma 3.16 that $s_{5} \in U$.

We shall show that, for each $i \in\{1,2,4\},\left|\sigma^{-1}\left[\left\{s_{i}\right\}\right]\right| \geq 3$. If $\left|\sigma^{-1}\left[\left\{s_{1}\right\}\right]\right|=2$, we can obtain an eight point triangle free subset of $Y$ as the union of $\sigma^{-1}\left[\left\{s_{1}\right\}\right]$ and a six point triangle free subset of $Y \backslash\left[\left\{s_{1}, s_{3}, s_{5}\right\}\right]$. The same argument shows that $\left|\sigma^{-1}\left[\left\{s_{2}\right\}\right]\right| \geq 3$ and $\left|\sigma^{-1}\left[\left\{s_{4}\right\}\right]\right| \geq 3$. However, this again requires $Y$ to have at least 13 points.

3.23 Theorem. $\lambda_{12}=8$.

Proof. Four disjoint triangles show that $\lambda_{12} \leq 8$. Suppose that we have some $Y \subseteq X$ with $|Y|=12$ and $\mu(Y) \leq 7$. By Lemma 3.21(a), there is at most one $y \in Y$ with $T_{y} \leq 1$. Thus, by Lemma 3.14, we may choose a triangle map $\sigma: Y \rightarrow S$ such that 
$\left\{s_{1}, s_{2}, s_{3}, s_{4}, s_{5}\right\} \subseteq \sigma[Y]$. By Lemma 3.22, we have $|\sigma[Y]| \leq 6$. Let

$$
U=\left\{s \in \sigma[Y]:\left|\sigma^{-1}[\{s\}]\right|=1\right\} .
$$

Assume first that $\sigma[Y]=\left\{s_{1}, s_{2}, s_{3}, s_{4}, s_{5}\right\}$. By Lemma 3.15, $\left\{s_{2}, s_{3}, s_{4}, s_{5}\right\} \cap U \neq \emptyset$, so assume without loss of generality that $s_{2} \in U$. Also

$$
Y \backslash \sigma^{-1}\left[\left\{s_{2}\right\}\right]=\sigma^{-1}\left[\left\{s_{3}\right\}\right] \cup \sigma^{-1}\left[\left\{s_{1}, s_{4}, s_{5}\right\}\right]
$$

so, by Lemma 3.21(b), $s_{3} \in U$. Since $\left\{s_{2}, s_{3}\right\} \subseteq U, \sigma$ can map at most one triangle into $\left\{s_{1}, s_{2}, s_{3}\right\}$. But then, if $\sigma\left(y_{2}\right)=s_{2}$ and $\sigma\left(y_{3}\right)=s_{3}$, we have $T_{y_{2}} \leq 1$ and $T_{y_{3}} \leq 1$, a contradiction.

Next assume that $\sigma[Y]=\left\{s_{1}, s_{2}, s_{3}, s_{4}, s_{5}, s_{8}\right\}$. By Lemma 3.15, $\left\{s_{2}, s_{3}, s_{4}, s_{5}\right\} \cap$ $U \neq \emptyset$. Assume without loss of generality that $s_{2} \in U$. But then $Y \backslash \sigma^{-1}\left[\left\{s_{2}\right\}\right]=$ $\sigma^{-1}\left[\left\{s_{1}, s_{4}, s_{5}\right\}\right] \cup \sigma^{-1}\left[\left\{s_{3}, s_{8}\right\}\right]$, contradicting Lemma 3.21(b).

We must have either $\sigma[Y]=\left\{s_{1}, s_{2}, s_{3}, s_{4}, s_{5}, s_{6}\right\}$ or $\sigma[Y]=\left\{s_{1}, s_{2}, s_{3}, s_{4}, s_{5}, s_{7}\right\}$. In either case, $\sigma[Y]$ is connected so by Lemma $3.17(b)$, there exist distinct $t_{1}, t_{2}, t_{3}, t_{4}$, $t_{5}$, and $t_{6}$ such that the triangles contained in $\sigma[Y]$ are exactly $\left\{t_{1}, t_{2}, t_{3}\right\},\left\{t_{1}, t_{4}, t_{5}\right\}$, and $\left\{t_{3}, t_{4}, t_{6}\right\}$. (See Diagram (c) above.) We claim that $\left\{t_{1}, t_{3}, t_{4}\right\} \cap U=\emptyset$. Suppose instead without loss of generality that $t_{1} \in U$. Then

$$
Y \backslash \sigma^{-1}\left[\left\{t_{1}\right\}\right]=\sigma^{-1}\left[\left\{t_{3}, t_{4}, t_{6}\right\}\right] \cup \sigma^{-1}\left[\left\{t_{4}, t_{5}\right\}\right],
$$

contradicting Lemma 3.21(b).

We now claim that if $i$ and $j$ are distinct members of $\{2,5,6\},\left\{t_{i}, t_{j}\right\} \subseteq U$, and $t_{l} \in C_{t_{i}} \cap C_{t_{j}}$, then $\left|\sigma^{-1}\left[\left\{t_{l}\right\}\right]\right| \geq 3$. Since $l \in\{1,3,4\}$ we know that $\left|\sigma^{-1}\left[\left\{t_{l}\right\}\right]\right| \geq 2$. Suppose $\left|\sigma^{-1}\left[\left\{t_{l}\right\}\right]\right|=2$. Then $\left|Y \backslash \sigma^{-1}\left[\left\{t_{l}, t_{i}, t_{j}\right\}\right]\right|=8$ so pick a six element triangle free subset $Z$ of $Y \backslash \sigma^{-1}\left[\left\{t_{l}, t_{i}, t_{j}\right\}\right]$. Then $Z \cup \sigma^{-1}\left[\left\{t_{i}, t_{j}\right\}\right]$ is triangle free.

By Lemma 3.15, $\left\{t_{1}, t_{3}, t_{5}, t_{6}\right\} \cap U \neq \emptyset,\left\{t_{1}, t_{2}, t_{4}, t_{6}\right\} \cap U \neq \emptyset$, and $\left\{t_{2}, t_{3}, t_{4}, t_{5}\right\} \cap U \neq$ $\emptyset$. Since $\left\{t_{1}, t_{3}, t_{4}\right\} \cap U=\emptyset$, we have that $\left|\left\{t_{2}, t_{5}, t_{6}\right\} \cap U\right| \geq 2$. Assume without loss of generality that $\left\{t_{5}, t_{6}\right\} \subseteq U$.

Since $\left\{t_{5}, t_{6}\right\} \subseteq U$ and $t_{4} \in C_{t_{5}} \cap C_{t_{6}}$, we have that $\left|\sigma^{-1}\left[\left\{t_{4}\right\}\right]\right| \geq 3$. We claim that $\sigma^{-1}\left[\left\{t_{4}\right\}\right]$ is a triangle. To see this, suppose first that $\left|\sigma^{-1}\left[\left\{t_{4}\right\}\right]\right| \geq 4$. Pick distinct $a, b$, and $c$ in $\sigma^{-1}\left[\left\{t_{4}\right\}\right]$ such that $\{a, b, c\}$ is not a triangle. If $t_{2} \notin U$, pick $x_{2} \neq y_{2}$ in $\sigma^{-1}\left[\left\{t_{2}\right\}, x_{3} \neq y_{3}\right.$ in $\sigma^{-1}\left[\left\{t_{3}\right\}\right.$, and $y_{5} \in \sigma^{-1}\left[\left\{t_{5}\right\}\right.$. Then $\left\{a, b, c, x_{2}, y_{2}, x_{3}, y_{3}, x_{5}\right\}$ is triangle free. Thus $t_{2} \in U$. Since $\left\{t_{2}, t_{5}\right\} \subseteq U$ and $t_{1} \in C_{t_{2}} \cap C_{t_{5}}$, we have that $\left|\sigma^{-1}\left[\left\{t_{1}\right\}\right]\right| \geq 3$. Since $\left\{t_{2}, t_{6}\right\} \subseteq U$ and $t_{3} \in C_{t_{2}} \cap C_{t_{6}}$, we have that $\left|\sigma^{-1}\left[\left\{t_{3}\right\}\right]\right| \geq 3$. But this contradicts the fact that $|Y|=12$.

Thus $\left|\sigma^{-1}\left[\left\{t_{4}\right\}\right]\right|=3$. Then $\left|Y \backslash \sigma^{-1}\left[\left\{t_{4}, t_{5}, t_{6}\right\}\right]\right|=7$ so pick a five element triangle free set $Z \subseteq Y \backslash \sigma^{-1}\left[\left\{t_{4}, t_{5}, t_{6}\right\}\right]$. If $\sigma^{-1}\left[\left\{t_{4}\right\}\right]$ is not a triangle, then $Z \cup \sigma^{-1}\left[\left\{t_{4}\right\}\right]$ is triangle free. Thus $\sigma^{-1}\left[\left\{t_{4}\right\}\right]$ is a triangle as claimed.

Pick $y_{5}$ and $y_{6}$ in $Y$ such that $\sigma\left(y_{5}\right)=t_{5}$ and $\sigma\left(y_{6}\right)=t_{6}$. We claim that $T_{y_{5}} \leq 2$ and $T_{y_{6}} \leq 2$. Suppose instead without loss of generality that $\left|T_{y_{5}}\right| \geq 3$. Then each of three triangles containing $y_{5}$ would be mapped onto $\left\{t_{1}, t_{4}, t_{5}\right\}$ and hence we would have the triangle $\sigma^{-1}\left[\left\{t_{4}\right\}\right] \subseteq C_{y_{5}}$, contradicting Theorem 3.9. 
Next we note that if $y \in \sigma^{-1}\left[\left\{t_{4}\right\}\right]$ and $T_{y} \geq 3$, then $y \in C_{y_{5}} \cap C_{y_{6}}$. (If $\{y, a, b\}$ and $\{y, c, d\}$ are triangles not contained in $\sigma^{-1}\left[\left\{t_{4}\right\}\right]$, then one is mapped onto $\left\{t_{3}, t_{4}, t_{6}\right\}$ and the other is mapped onto $\left\{t_{1}, t_{4}, t_{5}\right\}$.) Similarly, if $y \in \sigma^{-1}\left[\left\{t_{4}\right\}\right]$ and $T_{y}=2$, then $y \in C_{y_{5}} \cup C_{y_{6}}$.

Let $\sigma^{-1}\left[\left\{t_{4}\right\}\right]=\{u, v, w\}$. We claim that none of $u, v$, or $w$ is of degree 1. Suppose instead without loss of generality that $T_{u}=1$. Then by Lemma 3.21(a), $T_{v} \geq 2$ so $y_{5} \in C_{v}$ or $y_{6} \in C_{v}$. Assume without loss of generality that $y_{5} \in C_{v}$. Also $T_{y_{5}}=2$ so pick a triangle $A$ with $y_{5} \in A$ and $v \notin A$. Pick $z \in A \backslash\left\{y_{5}\right\}$ and pick a six point triangle free subset $Z$ of $Y \backslash\left\{u, v, y_{5}, z\right\}$. Then $Z \cup\left\{u, y_{5}\right\}$ is triangle free.

If we had two elements of $\{u, v, w\}$ of degree at least 3 , we would have $\{u, v, w\} \subseteq$ $C_{y_{5}}$ or $\{u, v, w\} \subseteq C_{y_{6}}$, contradicting Theorem 3.9. Thus we can assume without loss of generality that $T_{u}=T_{v}=2$. We claim that also $T_{w}=2$. So suppose that $T_{w} \geq 3$. Then $\left\{u, v, y_{5}, y_{6}\right\} \subseteq C_{w}$ so in $Y \backslash\{w\}, u, v, y_{5}$, and $y_{6}$ are all of degree 0 or 1, contradicting Lemma 3.20.

We cannot have $T_{y_{5}}=T_{y_{6}}=1$ by Lemma $3.21(a)$, so we assume without loss of generality that $T_{y_{5}}=2$. Then $\left|C_{y_{5}} \cap\{u, v, w\}\right|=2$ so without loss of generality $\{u, v\} \subseteq C_{y_{5}}$. Choose a six point triangle free subset $Z$ of $Y \backslash\left\{u, v, w, y_{5}\right\}$. Then $Z \cup\{u, v\}$ is triangle free.

3.24 Corollary. $\lambda_{11}=7$ and $\lambda_{13}=8$.

Proof. That $\lambda_{11}=7$ follows from Lemma 2.12 and the facts that $\lambda_{10}=6$ and $\lambda_{12}=8$. By Lemmas 2.12 and 3.5(a), we have that $8=\lambda_{12} \leq \lambda_{13} \leq \lambda_{3}+\lambda_{10}=8$.

It is interesting that for $\lambda_{3}, \lambda_{6}, \lambda_{9}$, and $\lambda_{12}$, the actual values are the trivial upper bounds obtained by considering disjoint triangles. We do not know whether $\lambda_{15}=10$ nor whether $\lambda_{18}=12$, but we do know that by Lemma 3.5(a), $\lambda_{21} \leq \lambda_{10}+\lambda_{11}=13$.

We have been able to obtain other results about the values of $\lambda_{k}$. We do not wish to burden the reader by providing the proofs of these, particularly as one or two of the proofs are very long. However, the reader may be interested to know that we have shown that $\lambda_{14}=9, \lambda_{17} \geq 10, \lambda_{20} \geq 11, \lambda_{23} \geq 12$ and $\lambda_{26} \geq 13$. In the case of $\lambda_{17}$ and $\lambda_{20}$, these estimates cannot differ from the correct value by more than 1 , because $\lambda_{17} \leq \lambda_{7}+\lambda_{10}=11$ and $\lambda_{20} \leq \lambda_{10}+\lambda_{10}=12$. These results can be used to prove that $\epsilon_{5}>\frac{1}{2}$. Thus all of our results are consistent with the conjecture that $\epsilon_{n}>\frac{1}{2}$ for every $n$. 


\section{Values of $\epsilon_{k}$}

We begin by noting that our knowledge of values of $\lambda_{k}$ provides some information about low values of $\epsilon_{k}$.

4.1 Lemma. $\epsilon_{1}=1, \epsilon_{2}=\frac{2}{3}$, and $\epsilon_{3}=\frac{2}{3}$.

Proof. The first two assertions are trivial, while the third conclusion follows from the fact that $\left|X_{1,4}\right|=8$ and the known values of $\lambda_{k}$ for $k \in\{1,2, \ldots, 8\}$.

We now set out to show that $\epsilon_{4}=.6$.

4.2 Lemma. For any $Y \subseteq X_{1,5}$ with $|Y| \neq 14, \frac{\mu(Y)}{|Y|} \geq \frac{3}{5}$.

Proof. From the values of $\lambda_{k}$ obtained in the last section, we know that, if $Y$ is a subset of $X_{1,5}$ and $\frac{\mu(Y)}{|Y|}<\frac{3}{5}$, then $|Y| \geq 14$. On the other hand, let

$$
\begin{aligned}
Z=\{ & (\{1,2\},\{5\}),(\{1\},\{2,5\}),(\{1,3\},\{5\}),(\{1\},\{3,5\}),(\{1,2,3\},\{5\}), \\
& (\{1\},\{2,3,5\}),(\{1,4\},\{5\}),(\{1\},\{4,5\}),(\{1,2,4\},\{5\}),(\{1\},\{2,4,5\}), \\
& (\{1,3,4\},\{5\}),(\{1\},\{3,4,5\}),(\{1,2,3\},\{4,5\}),(\{1,2\},\{3,4,5\})\} .
\end{aligned}
$$

A routine check shows that $Z$ contains no triangles. Since any $n$-element subset of $X_{1,5}$ includes at least $n-6$ members of $Z$, we have that for any $Y \subseteq X_{1,5}$ with $|Y|=n$, $\mu(Y) \geq \frac{n-6}{n}$. Now $\frac{n-6}{n} \geq \frac{3}{5}$ if $15 \leq n \leq 20$.

We shall now show that, for any $Y \subseteq X_{1,5}$ with $|Y|=14, \mu(Y) \geq 9$. We have, in fact, been able to prove this for any $Y \subseteq X$. However, the proof is much shorter for a subset of $X_{1,5}$. It will be convenient to regard $X_{1,5}$ as embedded in $\{-1,0,1\}^{3}$ by the mapping $x \mapsto\left(\pi_{2}(x), \pi_{3}(x), \pi_{4}(x)\right)$.

4.3 Lemma. Suppose that $Y \subseteq X_{1,5}$ and that $|Y|=14$. Then $\mu(Y) \geq 9$.

Proof. Assume that $\mu(Y) \leq 8$.

We observe that $Y$ is connected, since, for any $m_{1}, m_{2} \in \mathbb{N}, m_{1}+m_{2}=14$ implies that $\lambda_{m_{1}}+\lambda_{m_{2}} \geq 9$. Furthermore, if $m_{1}+m_{2}=13, \lambda_{m_{1}}+\lambda_{m_{2}} \geq 9$ unless $\left\{m_{1}, m_{2}\right\}=$ $\{3,10\}$. So a thirteen point subset of $Y$ cannot be expressed as the union of two disconnected sets unless one of these is a triangle.

We also observe that $Y$ contains no points of degree 1. If $y \in Y$ had degree 1, we could choose a point $z \neq y$ in the triangle containing $y$, and then obtain a nine point triangle free set in $Y$ by adding $y$ to an eight point triangle free subset of $Y \backslash\{y, z\}$.

We define a triangle map $\sigma: X_{1,5} \rightarrow S$ by putting $\sigma(x)=\left(\pi_{3}(x), \pi_{4}(x)\right)$. Since $Y$ can have at most 8 points in the triangle free subset $Z$ defined in Lemma $4.2, X_{1,5} \backslash Z \subseteq$ $Y$. It follows that $\left\{s_{1}, s_{2}, s_{3}, s_{4}, s_{5}, s_{8}\right\} \subseteq \sigma[Y]$. Since $\sigma[Y]$ is connected, $s_{6} \in \sigma[Y]$.

Let $U=\left\{s \in S:\left|\sigma^{-1}[\{s\}] \cap Y\right|=1\right\}$. We shall show that $s_{6}, s_{2} \notin U$. To see this, suppose that $s_{6} \in U$ and that $\sigma^{-1}\left[\left\{s_{6}\right\}\right] \cap Y=\{y\}$. Since $Y \backslash\{y\}$ is a thirteen point set which is the disconnected union of $Y \cap \sigma^{-1}\left[\left\{s_{8}\right\}\right]$ and $Y \cap \sigma^{-1}\left[S \backslash\left\{s_{6}, s_{8}\right\}\right], Y \cap \sigma^{-1}\left[\left\{s_{8}\right\}\right]$ must be a triangle. Suppose that $Y \cap \sigma^{-1}\left[\left\{s_{8}\right\}\right]=\left\{u_{1}, u_{2}, u_{3}\right\}$. For each $i \in\{1,2,3\}, u_{i}$ belongs to at least two triangles in $Y$ and one of these must be mapped to $\left\{s_{2}, s_{6}, s_{8}\right\}$ 
by $\sigma$, and must therefore contain $y$. So $\left\{u_{1}, u_{2}, u_{3}\right\} \subseteq C_{y}$, contradicting Theorem 3.9. So $s_{6} \notin U$, and the same argument shows that $s_{2} \notin U$.

Note that $\left|\sigma^{-1}\left[\left\{s_{i}\right\}\right]\right|=3$ for $i \in\{1,4,8\}$ and $\left|\sigma^{-1}\left[\left\{s_{i}\right\}\right]\right|=2$ for $i \in\{3,6,7\}$. We shall show that there are two distinct values of $i \in\{1,4,8\}$ for which $\left|\sigma^{-1}\left[\left\{s_{i}\right\}\right] \cap Y\right|=3$. Suppose on the contrary that there are distinct $i, j \in\{1,4,8\}$ for which there exist $x_{i} \in$ $\sigma^{-1}\left[\left\{s_{i}\right\}\right] \backslash Y$ and $x_{j} \in \sigma^{-1}\left[\left\{s_{j}\right\}\right] \backslash Y$. We can form a thirteen point triangle free subset $Z^{\prime}$ of $X_{1,5}$ as follows. We adjoin two points in each set $\sigma^{-1}\left[\left\{s_{k}\right\}\right]$ for $k \in\{1,3,4,6,7,8\} \backslash$ $\{i, j\}$ to $\left(\sigma^{-1}\left[\left\{s_{i}\right\}\right] \backslash\left\{x_{i}\right\}\right) \cup\left(\sigma^{-1}\left[\left\{s_{j}\right\}\right] \backslash\left\{x_{j}\right\}\right)$. We also adjoin $(1,0,0)$, which belongs to a unique triangle in $X_{1,5}$, namely $\{(-1,0,0),(0,0,0),(1,0,0)\}=\sigma^{-1}\left[\left\{s_{2}\right\}\right]$. Since $\left\{x_{i}, x_{j}\right\} \subseteq X_{1,5} \backslash\left(Y \cup Z^{\prime}\right)$, we must have $\left|Y \cap Z^{\prime}\right| \geq 9$, a contradiction.

Put $Y_{0}=\sigma^{-1}\left[\left\{s_{2}, s_{6}, s_{8}\right\}\right] \cap Y, Y_{1}=\sigma^{-1}\left[\left\{s_{1}, s_{4}, s_{5}\right\}\right] \cap Y, m_{0}=\left|Y_{0}\right|$ and $m_{1}=$ $\left|Y_{1}\right|$. Note that $Y_{0}$ and $Y_{1}$ are disconnected. Now $m_{0} \geq 5$, because $s_{2}, s_{6} \notin U$, and $m_{1} \geq 5$, because $\left|\sigma^{-1}\left[\left\{s_{1}\right\}\right] \cap Y\right|=3$ or $\left|\sigma^{-1}\left[\left\{s_{4}\right\}\right] \cap Y\right|=3$. Furthermore, either $\left|\sigma^{-1}\left[\left\{s_{8}\right\}\right] \cap Y\right|=3$ and therefore $m_{0} \geq 7$, or $\left|\sigma^{-1}\left[\left\{s_{1}\right\}\right] \cap Y\right|=3=\left|\sigma^{-1}\left[\left\{s_{4}\right\}\right] \cap Y\right|$ and $m_{1} \geq 7$. So $m_{0} \geq 5$ and $m_{1} \geq 7$, or vice-versa. Since $\lambda_{5}+\lambda_{7}=9$, it follows that $\mu(Y) \geq 9$.

4.4 Theorem. $\epsilon_{4}=\frac{3}{5}$.

Proof. We saw in the proof of Corollary 3.19 that there is a ten element subset of $X_{1,5}$ which has no seven element triangle free subset. Consequently, $\epsilon_{4} \leq \frac{6}{10}$. Our claim now follows from Lemmas 4.2 and 4.3 .

By virtue of Lemma 2.12, we have that $\lim _{k \rightarrow \infty} \epsilon_{k}$ exists. Question 1.3 asks precisely whether $\lim _{k \rightarrow \infty} \epsilon_{k}>0$. As we have previously remarked, we do not know whether $\lim _{k \rightarrow \infty} \epsilon_{k}<\frac{1}{2}$. What are able to show is that, while the sequence $\left\langle\epsilon_{k}\right\rangle_{k=1}^{\infty}$ may approach 0 , it at least does so slowly.

4.5 Lemma. Let $k \in \mathbb{N} \backslash\{1\}$ and let $W \subseteq X_{1, k+1}$ such that for all $(F, G) \in W, k \notin F$. Then there exists $Z \subseteq W$ such that $Z$ contains no triangles and $|Z| \geq \epsilon_{k-1} \cdot|W|$.

Proof. Let $A=\{(F, G) \in W: k \notin G\}$ and let $B=\{(F, G) \in W: k \in G\}$. Define $\psi: A \rightarrow X_{1, k}$ and $\tau: B \rightarrow X_{1, k}$ by $\psi(F, G)=(F,(G \backslash\{k+1\}) \cup\{k\})$ and $\tau(F, G)=$ $(F, G \backslash\{k+1\})$. Then $\psi$ and $\tau$ are injective and take triangles to triangles, so one may choose $Z_{1} \subseteq A$ and $Z_{2} \subseteq B$ such that neither $Z_{1}$ nor $Z_{2}$ contains triangles, $\left|Z_{1}\right| \geq$ $\epsilon_{k-1} \cdot|A|$, and $\left|Z_{2}\right| \geq \epsilon_{k-1} \cdot|B|$. Any triangle contained in $A \cup B$ is necessarily contained in $A$ or contained in $B$, so $Z_{1} \cup Z_{2}$ contains no triangles and $\left|Z_{1} \cup Z_{2}\right| \geq \epsilon_{k-1} \cdot|A \cup B| . \square$

4.6 Lemma. Let $k \in \mathbb{N} \backslash\{1\}$, let $Y \subseteq X_{1, k+1}$ such that $\epsilon_{k}=\frac{\mu(Y)}{|Y|}$, and let $Q=$ $\{(F, G) \in Y: \max F=k\}$. Then

(a) $\epsilon_{k-1} \cdot|Y \backslash Q| \leq \epsilon_{k} \cdot|Y|$ and

(b) $|Q| \leq\left(\epsilon_{k}+\epsilon_{k} \cdot \epsilon_{k-1}-\epsilon_{k-1}\right) \cdot|Y|$.

Proof. (a). Pick by Lemma 4.5 some $Z \subseteq Y \backslash Q$ such that $Z$ contains no triangles and $|Z| \geq \epsilon_{k-1} \cdot|Y \backslash Q|$. Then $\epsilon_{k-1} \cdot|Y \backslash Q| \leq|Z| \leq \mu(Y)=\epsilon_{k} \cdot|Y|$. 
(b). If $Q=\emptyset$, the conclusion is trivial, so we assume that $Q \neq \emptyset$. Let $U=$ $\{(F, G) \in Y: G=\{k+1\}\}$ and note that $Q \subseteq U$. By Lemma 4.5 pick $Z \subseteq Y \backslash U$ such that $|Z| \geq \epsilon_{k-1} \cdot|Y \backslash U|$ and $Z$ contains no triangles. Notice also that $U$ contains no triangles, so that $|U| \leq \mu(Y)=\epsilon_{k} \cdot|Y|$.

Now $Q \cup Z$ contains no triangles, so $|Q \cup Z| \leq \mu(Y)=\epsilon_{k} \cdot|Y|$. Let $\delta=\frac{|Q|}{|U|}$. Then we have $\epsilon_{k-1} \cdot|Y \backslash U|+\delta \cdot|U| \leq|Z|+|Q|=|Z \cup Q| \leq \epsilon_{k} \cdot|Y|$. Therefore, $|Q|=\delta \cdot|U| \leq \epsilon_{k} \cdot|Y|-\epsilon_{k-1} \cdot|Y|+\epsilon_{k-1} \cdot|U| \leq \epsilon_{k} \cdot|Y|-\epsilon_{k-1} \cdot|Y|+\epsilon_{k-1} \cdot \epsilon_{k} \cdot|Y|$.

4.7 Theorem. Let $k \in \mathbb{N} \backslash\{1\}$. Then

$$
\epsilon_{k} \geq \frac{\epsilon_{k-1}+\epsilon_{k-1}^{2}}{1+\epsilon_{k-1}+\epsilon_{k-1}^{2}} .
$$

Proof. Pick $Y \subseteq X_{1, k+1}$ such that $\epsilon_{k}=\frac{\mu(Y)}{|Y|}$. Let $Q=\{(F, G) \in Y: \max F=k\}$. Then by Lemma 4.6(a), $\epsilon_{k-1} \cdot|Y \backslash Q| \leq \epsilon_{k} \cdot|Y|$ so that $\epsilon_{k} \cdot|Y| \geq \epsilon_{k-1} \cdot|Y|-\epsilon_{k-1} \cdot|Q| \geq$ $\epsilon_{k-1} \cdot|Y|-\epsilon_{k-1} \cdot\left(\epsilon_{k}+\epsilon_{k} \cdot \epsilon_{k-1}-\epsilon_{k-1}\right) \cdot|Y|$ by Lemma $4.6(\mathrm{~b})$.

4.8 Corollary. For all $k \in \mathbb{N}, \epsilon_{k}>\frac{1}{\sqrt{2 k}}$.

\section{References}

[1] P. Erdős, J. Nešetríl, and V. Rödl, On Pisier type problems and results (combinatorial applications to Ramsey Theory), in Mathematics of Ramsey Theory, J. Nešetríl, and V. Rödl eds., Springer-Verlag, Berlin, (1990), 214-231.

[2] P. Erdős, J. Nešetríl, and V. Rödl, A remark on Pisier type theorems, Congr. Numer. 113 (1996), 101-109.

[3] K. Milliken, Ramsey's Theorem with sums or unions, J. Comb. Theory (Series A) 18 (1975), 276-290.

[4] J. Nešetríl, and V. Rödl, Partite construction and Ramseyan theorems for sets, numbers and spaces, Comment. Math. Univ. Carolinae 28 (1987), 569-580.

[5] G. Pisier, Arithmetic characterizations of Sidon sets, Bull. Amer. Math. Soc. 8 (1983), 87-89.

[6] K. Roth, On certain sets of integers, J. London Math. Soc. 28 (1953), 104-109.

[7] K. Roth, On certain sets of integers, II, J. London Math. Soc. 29 (1954), 20-26.

[8] E. Szemerédi, On sets of integers containing no $k$ elements in arithmetic progression, Acta. Math. 27 (1975), 199-245.

[9] A. Taylor, A canonical partition relation for finite subsets of $\omega$, J. Comb. Theory (Series A) 21 (1976), 137-146. 